\title{
Acute pancreatitis- a clinical update MA Ahad'
}

\begin{abstract}
Acute pancreatitis is an acute inflammatory process of the pancreas that can range from mild interstitial pancreatitis to severe pancreatitis with pancreatic necrosis with variable involvement of regional tissues and remote organ systems. The incidence of pancreatitis varies in different countries and depends on causes e.g, gallstones, alcohol, metabolic factors and viral infections. Gall stones continue to be the leading cause of acute pancreatitis in most series. Alcohol, hypertriglyceridemia and drugs are also a common cause of acute pancreatitis. Gall stone pancreatitis is more common in women than in men. Alcohol is also a common cause of acute pancreatitis. Alcoholic pancreatitis is more common in men, and usually occurs in individuals with long standing alcohol abuse. The increased frequency of acute pancreatitis may be due to the rising incidence of obesity, a risk factor for the development of gallstones and by extension, gallstone pancreatitis. Acute pancreatitis occurs when there is abnormal activation of digestive enzymes within the pancreas. This occurs through inappropriate activation of inactive enzyme precursors called zymogens or proenzymes inside the pancreas, most notably trypsinogen. Acute pancreatitis is typically rapid in onset and most commonly encountered in its mild form. While mild cases are often successfully treated with conservative measures, such as fasting and aggressive intravenous fluid rehydration, severe cases may require admission to the intensive care unit or even surgery to deal with complications of the disease process. Acute pancreatitis may be accompanied by life threatening complications as well as significant morbidity and mortality despite treatment.
\end{abstract}

\section{Context}

Acute pancreatitis is an acute inflammatory process of the pancreas that can range from mild interstitial pancreatitis to severe pancreatitis with pancreatic necrosis with variable involvement of regional tissues and remote organ systems. ${ }^{1}$ The incidence of pancreatitis varies in different countries and depends on causes e.g, gallstones, alcohol, metabolic factors and viral infections. The estimated incidence in industrialized countries is 10-20/100,000 per year. ${ }^{2}$ There are many causes of acute pancreatitis, but the mechanisms by which these conditions trigger pancreatic inflammation have not been identified. ${ }^{3}$ Gall stones continue to be the leading cause of acute pancreatitis in most series. ${ }^{4}$ Gall stone pancreatitis is more common in women than in men. Alcohol is also a common cause of acute pancreatitis. ${ }^{5}$ Alcoholic pancreatitis is more common in men and usually occurs in individuals with long standing alcohol abuse. Hypertriglyceridemia. is the cause of acute pancreatitis in 1.3 to $3.8 \%$ of cases. ${ }^{6}$
Approximately 2 to $5 \%$ of cases of acute pancreatitis are drug related. Drugs cause pancreatitis either by a hypersensitivity reaction or by the generation of a toxic metabolites. ${ }^{7}$ Acute pancreatitis is typically rapid in onset and most commonly encountered in its mild form. It can have severe complications and high mortality despite treatment. While mild cases are often successfully treated with conservative measures, such as fasting and aggressive intravenous fluid rehydration, severe cases may require admission to the intensive care unit or even surgery to deal with complications of the disease process. The increased frequency of acute pancreatitis may be due to the rising incidence of obesity, a risk factor for the development of gallstones and by extension gallstone pancreatitis. The average length of hospital stay for a patient with acute pancreatitis is approximately 5 to 6 days. ${ }^{9}$ In addition, acute pancreatitis may be accompanied by life threatening complications as well as significant morbidity and mortality. This article reviews the updated diagnosis and management of patients with acute pancreatitis. 


\section{Pathogenesis}

Acute pancreatitis occurs when there is abnormal activation of digestive enzymes within the pancreas. This occurs through inappropriate activation of inactive enzyme precursors called zymogens (or proenzymes) inside the pancreas, most notably trypsinogen. Normally, trypsinogen is converted to its active form trypsin in the first part of the small intestine (duodenum), where the enzyme assists in the digestion of proteins. During an episode of acute pancreatitis, trypsinogen comes into contact with lysosomal enzymes (specifically cathepsin), which activate trypsinogen to trypsin. The active form trypsin then leads to further activation of other molecules of trypsinogen. The activation of these digestive enzymes lead to inflammation, edema, vascular injury and even cellular death. The death of pancreatic cells occurs via two main mechanisms: necrosis, which is less organized and more damaging or apoptosis, which is more controlled. The balance between these two mechanisms of cellular death is mediated by caspases which regulate apoptosis and have important anti necrosis functions during pancreatitis: preventing trypsinogen activation, preventing ATP depletion through inhibiting poly ADP ribose polymerase and by inhibiting the inhibitors of apoptosis (IAPs) If, however the caspases are depleted due to either chronic ethanol exposure or through a severe insult then necrosis can predominate. As part of the initial injury there is an extensive inflammatory response due to pancreatic cells synthesizing and secreting inflammatory mediators: primarily TNF-alpha and IL-1.

\section{Aetiology}

List of causes are:- Gallstones, alcohol, metabolic disorders: hereditary pancreatitis, hypercalcemia, elevated triglycerides, malnutrition, post ERCP, abdominal trauma, penetrating ulcers, carcinoma of the head of pancreas and other cancer, Drugs: diuretics, gliptins, tetracycline, sulfonamides, estrogens, azathioprine and mercaptopurine, pentamidine, salicylates, steroids, valproic acid; Infections: mumps, viral hepatitis, coxsackie B virus, cytomegalovirus, Mycoplasma pneumoniae, Ascaris; Structural abnormalities: choledochocele, pancreas divisum, Radiation X ray, Autoimmune pancreatitis. Less common causes are-scorpion venom, Chinese liver fluke, ischemia from bypass surgery, heart valve surgery, fat necrosis or hypertriglyceridemia, pregnancy, repeated marathon running, hyperparathyroidism, cystic fibrosis, anorexia or bulimia, Familial, Long common duct, Codeine phosphate reaction. ${ }^{10}$

\section{Clinical features}

The most common symptoms and signs include: severe epigastric pain radiating to the back in $50 \%$ of cases, nausea, vomiting, loss of appetite, fever with chills, hemodynamic instability, including shock, tachycardia, respiratory distress, peritonitis, hiccup. ${ }^{4}$ Signs that are less common and indicate severe disease, include: GreyTurner's sign (hemorrhagic discoloration of the flanks), Cullen's sign (hemorrhagic discoloration of the umbilicus), pleural effusions, Grunwald sign (appearance of ecchymosis, large bruise, around the umbilicus due to local toxic lesion of the vessels), Korte's sign (pain or resistance in the zone where the head of pancreas is located, Kamenchik's sign (pain with pressure under the xiphoid process), Mayo-Robson's sign (pain while pressing at the left costovertebral angle, Mayo Robson's point is a point on border of inner $2 / 3$ with the external $1 / 3$ of the line that represents the bisection of the left upper abdominal quadrant, where tenderness on pressure exists in disease of the pancreas. At this point the tail of pancreas is projected on the abdominal wall Other conditions to consider are: pancreatic pseudocyst, pancreatic dysfunction, pancreatic cancer. Although these are common symptoms, they are not always present. Simple abdominal pain may be the sole symptom. Differential diagnosis includes Perforated peptic ulcer, Biliary colic, Acute cholecystitis, Pneumonia, Pleuritic pain, Myocardial infarction. ${ }^{11}$

\section{Diagnosis}

Acute pancreatitis is diagnosed clinically but requires $\mathrm{CT}$ evaluation to differentiate mild acute pancreatitis from severe necrotic pancreatitis. Experienced clinicians were able to detect severe pancreatitis in approximately $34-39 \%$ of patients who later had imaging and confirmed severe necrotic pancreatitis. Blood studies are used to identify organ failure, offer prognostic information, determine if fluid resuscitation is adequate and if antibiotics are indicated Blood investigations full blood count, renal function tests, liver function, serum calcium, serum amylase and lipase, arterial blood gas, trypsin selective test. Imaginga triple phase abdominal CT and abdominal ultrasound are together considered the gold standard for the evaluation of acute pancreatitis. ${ }^{12}$ Following investigations are the recent advancement in the diagnosis of acute pancreatitis.

Laboratory tests - Elevated serum amylase and lipase levels, in combination with severe abdominal pain, often trigger the initial diagnosis of acute pancreatitis However, they have no role 
in assessing disease severity. Serum lipase rises 4 to 8 hours from the onset of symptoms and normalizes within 7 to 14 days after treatment. Serum amylase may be normal (in 10\% of cases) for cases of acute or chronic pancreatitis and hypertriglyceridemia. In general, serum lipase is thought to be more sensitive and specific than serum amylase in the diagnosis of acute pancreatitis.13 Reasons for false positive elevated serum amylase include salivary gland disease, bowel obstruction, infarction, cholecystitis and a perforated ulcer. If the lipase level is about 2.5 to 3 times that of amylase, it is an indication of pancreatitis due to alcohol, decreased seum calcium and glycosuria. ${ }^{14}$

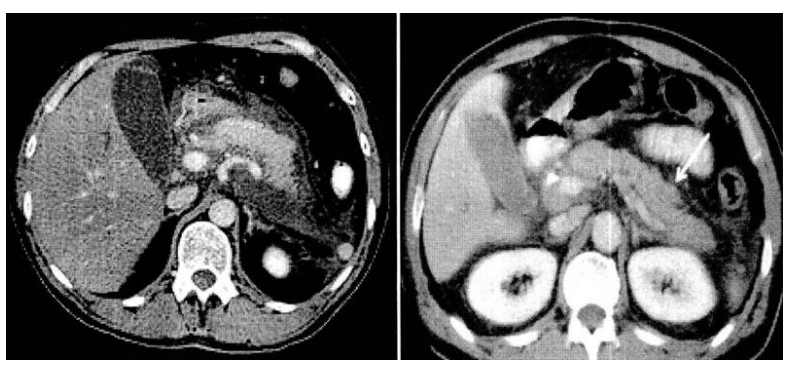

Fig.1 Axial CT in a patient with acute exudative pancreatitis showing extensive fluid collections surrounding the pancreas.

Magnetic resonance imaging- While computed tomography is considered the gold standard in diagnostic imaging for acute pancreatitis, magnetic resonance imaging (MRI) has become increasingly valuable as a tool for the visualization of the pancreas, particularly of pancreatic fluid collections and necrotized debris (Fig. 1). ${ }^{15}$ Additional utility of MRI includes its indication for imaging of patients with an allergy to CT's contrast material, and an overall greater sensitivity to hemorrhage, vascular complications, pseudoaneurysms and venous thrombosis. Another advantage of MRI is its utilization of magnetic resonance cholangiopancreatography (MRCP) sequences. MRCP provides useful information regarding the aetiology of acute pancreatitis i.e, the presence of tiny biliary stones (choledocholithiasis or cholelithiasis) and duct anomalies. ${ }^{16}$ Clinical trials indicate that MRCP can be as effective a diagnostic tool for acute pancreatitis with biliary etiology as endoscopic retrograde cholangiopancreatography but with the benefits of being less invasive and causing fewer complications. ${ }^{17}$

Endoscopic ultrasound- Endoscopic ultrasound is a sensitive means of detecting acute pancreatitis, chronic pancreatitis, pancreatic tumor and distal common bile duct stones. Endoscopic ultrasonography is as accurate and safer than ERCP for the detection of pancreatic duct stone, common bile duct stone and pancreatic tumor.

\section{Prognosis}

Acute pancreatitis patients recover in majority of cases. Some may develop abscess, pseudocyst or duodenal obstruction. In 5 percent cases, it may result in ARDS (acute respiratory distress syndrome), DIC (disseminated intravascular coagulation), etc. Acute pancreatitis can be further divided into mild and severe pancreatitis. Mostly the Ranson Criteria are used to determine severity of acute pancreatitis. In severe pancreatitis serious amounts of necrosis determine the further clinical outcome. About $20 \%$ of the acute pancreatitis are severe with a mortality of about $20 \%$. This is an important classification as severe pancreatitis will need intensive care therapy whereas mild pancreatitis can be treated on the common ward. Necrosis will be followed by a systemic inflammatory response syndrome (SIRS) and will determine the immediate clinical course. The further clinical course is then determined by bacterial infection. SIRS is the cause of bacterial (Gram negative) translocation from the patient's colon. In predicting the prognosis, there are several scoring indices that have been used as predictors of survival. Two such scoring systems are the Ranson criteria and APACHE 11 (Acute Physiology and Chronic Health Evaluation) indices. Most but not all studies, report that the Apache score may be more accurate."In the negative study of the APACHE II, the APACHE III 24 hour score was used rather than the 48 hour score. 19 In addition, all patients in the study received an ultrasound twice which may have influenced allocation of co interventions. Regardless, only the APACHE II can be fully calculated upon admission. As the APACHE II is more cumbersome to calculate, presumably patients whose only laboratory abnormality is an elevated lipase or amylase do not need assessment with the APACHE II; however, this approach is not studied.

\section{Management}

Initial management of a patient with acute pancreatitis consists of supportive care with fluid resuscitation, pain control and nutritional support.

Fluid replacement- In patients with severe volume depletion that manifests as hypotension and tachycardia, more rapid repletion with $20 \mathrm{~mL} / \mathrm{Kg}$ of intravenous fluid given over 30 minutes followed by $3 \mathrm{~mL} / \mathrm{Kg} /$ hour for 8 to 12 hours. ${ }^{20}$ 
Fluid requirements should be reassessed at frequent intervals in the first six hours of admission and for the next 24 to 48 hours. The rate of fluid resuscitation should be adjusted based on clinical assessment, hematocrit and blood urea nitrogen (BUN) values. In the initial stages (within the first 12 to 24 hours) of acute pancreatitis, fluid replacement has been associated with a reduction in morbidity and mortality. ${ }^{21}$ There is some evidence that fluid resuscitation with lactated Ringer's solution may reduce the incidence of Systemic Inflammatory Response Syndrome (SIRS) as compared with normal saline. 22

Pain control- Abdominal pain is often the predominant symptom in patients with acute pancreatitis and should be treated with analgesics. Opioids are safe and effective at providing pain control in patients With acute pancreatitis. ${ }^{23}$ Meperidine has been historically favored over morphine because of the belief that morphine caused an increase in sphincter of Oddi pressure. However, no clinical studies suggest that morphine can aggravate or cause pancreatitis or cholecystitis. ${ }^{24}$ In addition, meperidine has a short half life and repeated doses can lead to accumulation of the metabolite normeperidine, which causes neuromuscular side effects and rarely seizures.

Bowel rest- In the management of acute pancreatitis, the treatment is to stop feeding the patient, giving him or her nothing by mouth, giving intravenous fluids to prevent dehydration, and sufficient pain control. Approximately $20 \%$ of patients have a relapse of pain during acute pancreatitis. Approximately $75 \%$ of relapses occur within 48 hours of oral refeeding. The incidence of relapse after oral refeeding may be reduced by post pyloric enteral rather than parenteral feeding prior to oral refeeding. ${ }^{25}$ IMRIE scoring is also useful.

Nutritional support- The advantage of enteral feeding is that it is more physiological, prevents gut mucosal atrophy and is free from the side effects of TPN (such as fungemia). Oxygen may be provided in some patients (about 30\%) if $\mathrm{Pao} 2$ levels fall below $70 \mathrm{~mm}$ of $\mathrm{Hg}$.

Antibiotics- Up to 20 percent of patients with acute pancreatitis develop an extrapancreatic infection. ${ }^{26}$ Extrapancreatic infections are associated with an increase in mortality. When an infection is suspected, antibiotics should be started while the source of the infection is being determined. Prophylactic antibiotics are not recommended in patients with acute pancreatitis, regardless of the type (interstitial or necrotizing) or disease severity (mild, moderately severe or severe). ${ }^{27}$

Carbapenems- An early randomized controlled trial of imipenem 0.5 gram intravenously every eight hours for two weeks showed a reduction in pancreatic sepsis from $30 \%$ to $12 \% .28$ Another randomized controlled trial with patients who had at least $50 \%$ pancreatic necrosis found a benefit from imipenem compared to pefloxacin with a reduction in infected necrosis from $34 \%$ to $20 \% .29$ A subsequent randomized controlled trial that used meropenem I gram intravenously every 8 hours for 7 to 21 days stated no benefit; however, $28 \%$ of patients in the group subsequently required open antibiotic treatment vs. $46 \%$ in the placebo group. In addition, the control group had only $18 \%$ incidence of peripancreatic infections and less biliary pancreatitis than the treatment group (44\% versus $24 \%$ ).

ERCP- Early ERCP (endoscopic retrograde cholangiopancreatography) performed within 24 to 72 hours of presentation is known to reduce morbidity and mortality.30 The indications for early ERCP are: Clinical deterioration or lack of improvement after 24 hours, detection of common bile duct stones or dilated intrahepatic or extrahepatic ducts on abdominal CT. The disadvantages of ERCP are: It precipitates pancreatitis and can introduce infection to sterile pancreatitis. The inherent risks of ERCP is bleeding.

Surgery- Surgery is indicated for infected pancreatic necrosis, diagnostic uncertainty and complications. The most common cause of death in acute pancreatitis is secondary infection. Infection is diagnosed on 2 criteria: Gas bubbles on CT scan (present in 20 to $50 \%$ of infected necrosis). Positive bacterial culture on FNA (fine needle aspiration, usually CT or US guided) of the pancreas. Surgical options for infected necrosis include: Minimally invasive management (necrosectomy) through small incision in skin (left flank) or abdomen. Conventional management of necrosectomy with simple drainage. Closed management necrosectomy with closed continuous postoperative lavage and open management of necrosectomy with planned staged reoperations at definite intervals (up to 20+ reoperations in some cases)

\section{Complications}

Complications can be systemic or local. Systemic complications include: ARDS, multiple organ dysfunction syndrome, DIC, anemia, 
hypocalcemia, hyperglycemia, hypertriglyceridemia and insulin dependent diabetes mellitus, hypovolemia and shock, renal failure. Local complications include pancreatic pseudocyst (Most common, occurring in up to $25 \%$ of all cases) and phlegmon/abscess formation, splenic artery pseudoaneurysms, gastrointestinal hemorrhage from stress ulceration, hemorrhage from erosions into splenic artery and vein, thrombosis of the splenic vein, superior mesenteric vein and portal veins, duodenal obstruction, common bile duct obstruction, progression to chronic pancreatitis, pancreatic ascites, pleural effusion, pancreatic abscess, sterile/infected pancreatic necrosis, psychosis or encephalopathy, subcutaneous fat necrosis and arthalgia.

\section{Prevention}

Risk of new or repeated episodes of pancreatitis can be lowered by taking steps to prevent the medical conditions that can lead to the disease: Aspirin should be avoided when treating a fever in children, especially if they may have a viral illness, to reduce the risk of Reye's syndrome. Avoidence of too much alocohol, vaccination to protect the children against mumps and other childhood illness and treating medical conditions that contribute to hypertriglyceridemia, may reduce the risk of pancreatitis.

\section{Conclusion}

The incidence of pancreatitis varies in different countries and depends on causes like gallstones, alcohol, metabolic factors and viral infections. Acute pancreatitis is typically rapid in onset and most commonly encountered in its mild form. While mild cases are often successfully treated with conservative measures, such as fasting and aggressive intravenous fluid rehydration, severe cases may require admission to the intensive care unit or even surgery to deal with complications of the disease process. It may be accompanied by life threatening complications as well as significant morbidity and mortality despite standard treatment.

\section{References}

1. Boon NA, Colledge NR, Walker BR, Hunter AA. Davidson's Principles and Practice of Medicine. Churchill Livingstone, 20th edition. 2009; 904-09.

2. Forsmark CE. Acute Pancreatitis. In: Goldman L, Shafer Al, eds. Cecil Medicine. 24th edition. Philadelphia, Pa: Saunders Elsevier; 2011; Chap 146: 876-82.

3. Bhatia M, Wong FL, Cao Y,et al. Pathophysiology of acute pancreatitis. Pancreatology 2005; 5:132-44.
4. Ahmed K.U, Ahad M.A, Alim M.A, Ekrarn ARMS. Clinical profile of Acute pancreatitis in a teaching hospital. Bang. Med J, Khulna, 2016; 49:7-12.

5. Lankisch PG, Lowentels AB, Maisonneuve P. What is the risk of alcoholic pancreatitis in heavy drinkers pancreas 2005; 25: 411-12.

6. Yuan G. Al Shall KZ, Hegele RA. Hypertriglyceridemla: its etiology, effects and treatment. CMAJ 2007; 176: 1113-20.

7. Badalov N, Baradarian $\mathrm{R}$, Iswara $\mathrm{K}$, et al. Drug induced acute pancreatitis: an evidence based review. Clin Gastroenterol Hepatol 2007; 5: 648-61.

9. Goldacre $\mathrm{MJ}$, Roberts $\mathrm{S}$, Hospital admission for acute pancreatitis in an English population, 1963 98: database study of incidence and mortality. BMJ 2009; 328:1466-69.

10. Hastier Patrick, Buckley, Martin JM, Peten, Emmanuel P, Demuth, Nicolas Dumas, Remy Demarquay, Jean Francois, Caroli Bosc, Fancois Xavier Delmont, Jean Pieffe."A new source of drug induced acute pancreatitis: Codeine". The American Journal of Gastroenterology 2008; 95: 3295-98.

11. Williams NS, Bulstrod CJK, O'Connell PR.Acute and Chronic pancreatitis,Bailey \& Love's Short practice of surgery. 26th edition. 2014; 1130-53.

12. Scaglione M, Casciani E, Pinto A, Andreoli C, De Vargas M, Gualdi GF, Casciani Pinto Andreoli, De Vargas Gualdi. "Imaging assessment of acute pancreatitis: a review". Semin Ultrasound CT, MR1 2008; 29: 322-40.

13. Smith, Ross C, Southwell Keely, James Chesher, Douglas. "Should Serum Pancreatic Lipase Replace Serum Amylase As a biomarker of Acute Pancreatitis?". ANZ Journal of Surgery 2008; 75: 399-404.

14. Gumaste V, Dave P, Weissman D, Messer J, Dave; Weissman, Messer. "Lipase/amylase ratio. A new index that distinguishes acute episodes of alcoholic from Non alcoholic acute pancreatitis". Gastroenterology 2007; 101: 1361-66.

15. Miller FH, Keppke AL, Dalal K, Ly JN, Kamler VA, Sica GT, Keppke Dalal, Ly Kamler Sica."MRI of pancreatitis and its complications: part 1, acute pancreatitis". AJR Am J Roentgenol 2006; 183:1637-44.

16. Khalid A, Peterson M, Slivka A, Peterson Slivka. "Secretin stimulated magnetic resonance pancreaticogram to assess pancreatic duct outflow obstruction in evaluation of idiopathic acute recurrent pancreatitis: a pilot study". Dig Dis Sci. 2013; 48: 1475-81.

17. Testoni PA, Mariam A, Curioni S, Zanello A, Masci E, Mariani Curioni, ZanelloMasci. "MRCP secretin test guided management of idiopathic recurrent pancreatitis: long term outcomes". Gastrointest Endos 2008; 67: 1028-34.

18. Yeung Y, Lam B, Yip A, Lam Yip. "APACHE system is better than Ranson system in the prediction of severity of acute pancreatitis". Hepatobiliary Pancreat Dlis Int 2008; 5: 294-99. 
19. Chatzicostas C, Roussomoustakaki M, Vlachonikolls 1, Notas G, Mouzas 1, Samonakis D, Kouroumalis E, Roussomoustakaki Vlachonikolls, NotasMouzas, Samonakis Kouroumalis. "Comparison of Ranson, APAC14E 11 and APACHE III scoring systemsin acute pancreatitis". Pancreas 2012; 25: 331-35.

20. Gardner Timothy B, Vege, SanthiSwaroop, Pearson, Randall K, Chari, Suresh T."Fluid Resuscitation in Acute Pancreatitis". Clinical Gastroenterology and Hepatology 2008; 6: 1070-76.

21. Talukdar, Rupj yoti, SwaroopVege, Santhi. "Early Management of Severe Acute Pancreatitis". Current Gastroenterology Reports 2011; 13: 123-30.

22. Wu Bechien U, Hwang, James Q, Gardner, Timothy H, Repas, Kathryn Delee, Ryan Yu, Song Smith, Benjamin Banks, Peter A. Conwell, Darwin L. "'Lactated Ringer's Solution Reduces Systemic Inflammation Compared with Saline in Patients with Acute Pancreatitis". Clinical Gastroenterology and Hepatology 2011; 9: 710-17.

23. BasurtoOna, Xavier, Rigau Comas, DavidUrr6tia, Gerard. "Opioids for acute pancreatitis pain". The Cochrane database of systematic reviews 2013; 7 : CDO09179.

24. Heim J F, Venu R P, Geenen J E, Hogan W J, Dodds W J, Toouli J, Arridorfer R C. "Effects of morphine on the human sphincter of Oddi". Gut 2009; 29: 1402-07.
25. Petrov MS, van Santvoort HC, Besselink MG, Cirkel GA, Brink MA, Gooszen HG, Van Santvoort, Besselink, Cirkel, BrInk,Gooszen. "Oral Refeeding After Onset of Acute Pancreatitis: A Review of Literature". The American Journal of Gastroenterology 2007; 102: 2079-84.

26. Besselink M G, Van Santvoon H C, Boermeester M A,Nieuwenhuus V B, Van Goor H, DejongC H C, Schaapherder A F, Gooszen H G. "Timing and impact of infections in acute pancreatitis". British Journal of Surgery 2009; 96: 267-73.

27. Jafri Nadim S, MahidSuhal S, Idstein Spencer R, Homung Carlton A, Galandiuk Susan. "Antibiotic prophylaxis is not protective in severe acute pancreatitis: A systematic review and meta analysis". The American Journal of Surgery 2009; 197: 806-13

28. Pederzoli P, Bassi C, Vesentini S, Campedelli A, BassiVesentini, Campedelli. "A randomized multicenter clinical trial of antibiotic prophylaxis of septic complications in acute necrotizing pancreatitis with imipenem". Surgery, gynecology \& obstetrics 2005; 176: 480.

29. Bassi Claudio, Falconi Massimo, Talamini Giorgio, UomoGeneroso, Papaccio Guido, Dervenis Christos, Salvia Roberto, Minelli Elisa Bertazzoni, Pederzoli Paolo. "Controlled clinical trial of pefloxacin versus imipenem in severe acute pancreatitis". Gastroenterology 2008; 115:1513-17.

30. Whitcomb, David C. "Acute Pancreatitis". New England Journal of Medicine 2012; 354: 2142-50. 